\title{
Cutaneous lupus erythematosus flare following exposure to surgical light during a dental procedure
}

\author{
Janice Tiao, ${ }^{1,2}$ Victoria P Werth ${ }^{1,2}$
}

${ }^{1}$ Corporal Michael J. Crescenz VA Medical Center

${ }^{2}$ Department of Dermatology, University of Pennsylvania,

Philadelphia, PA, USA

\section{Correspondence to}

Dr Victoria Werth,

werth@mail.med.upenn.edu

Accepted 19 November 2015

\section{SUMMARY}

A patient in her 60 s with systemic lupus erythematosus presented to an outpatient dermatology clinic on multiple occasions, with exacerbations of cutaneous lupus after exposure to surgical lighting during dental procedures. Her photosensitivity to surgical lighting suggests that artificial light sources pose potential triggers of lupus erythematosus in extra photosensitive individuals. This case report summarises those potential triggers and some options to decrease exposure from surgical lighting.

\section{BACKGROUND}

It has been reported that up to $73 \%$ of patients with systemic lupus erythematosus (SLE) exhibit photosensitivity to ultraviolet radiation (UVR). ${ }^{1}$ Most descriptions of photosensitivity in SLE involve symptomatic exacerbation in response to direct sunlight exposure. ${ }^{2}$ However, fluorescent lights are increasingly recognised as a source of UVR that may trigger photosensitivity. ${ }^{3}$ We report a case of a patient meeting criteria for SLE whose acute cutaneous lupus erythematosus (ACLE) flared repeatedly following light exposure from surgical lighting during dental procedures.

\section{CASE PRESENTATION}

We present a case of a woman in her 60s who had been followed at the autoimmune skin diseases clinic at the University of Pennsylvania since 2003, for management of SLE and ACLE. She was seen at the clinic in May 2011 with a cutaneous lupus erythematosus (CLE) flare, which for her was an unusual occurrence due to her diligence about sun avoidance measures. At her visit, she reported a 5-day history of a pruritic, raised red rash initially beginning on her face and subsequently extending to her chest, back, arms and legs. Notably, 3 days prior to the onset of the rash, she had gone to her dentist for a dental crown, a procedure in which she was in the dentist's chair and exposed to surgical light for approximately $1 \mathrm{~h}$. On presentation to the clinic, she endorsed fatigue but was otherwise asymptomatic on review of systems. Her medications at the time of the visit were plaquenil $100 \mathrm{mg} /$ day, quinacrine $50 \mathrm{mg}$ every other day, azathioprine $150 \mathrm{mg} /$ day and prednisone $20 \mathrm{mg} /$ day, which had been increased from her baseline $7 \mathrm{mg} /$ day in response to the current flare. Physical examination was remarkable for erythaema on the face, chest, back, and upper and lower extremities, as well as livedo reticularis on the lower extremities.
In May 2014, the patient presented to the clinic with another rash concerning for a CLE flare. Five days prior to the onset of the rash, she had received another dental crown from the same dentist, who followed the same protocol as with her first crown. The rash began on her chest and neck and then extended to her back and lower extremities. At the time the rash began, she was taking azathioprine $175 \mathrm{mg} /$ day and prednisone $8.5 \mathrm{mg} /$ day. Physical examination in the clinic was notable for an eruption extending across her chest, back and upper and lower extremities.

The patient had been diagnosed with SLE in 1983. At the time of her diagnosis, the patient had been hospitalised for pericarditis and a plural effusion with accompanying joint pain. Other clinical symptoms over her long SLE history included fatigue, arthralgias, malar rash and photosensitivity. Abnormal laboratory tests included elevated antinuclear antibody tests, positive anti-double stranded DNA antibody tests, elevated anticardiolipin IgG antibodies, positive Sjögren syndrome antigen A (SSA)/Ro antibodies, and decreased C3 and C4 levels. Other findings suggestive of SLE included Raynaud's phenomenon and livedo reticularis in the setting of positive antiphospholipid antibodies; a biopsy of the patient's left leg showed organising intravascular thrombus of the deep arterials.

Management of the patient's fatigue and arthralgias involved low-dose prednisone from approximately $6-12 \mathrm{mg} /$ day with increases as necessary with flares. Attempts to decrease prednisone below this level, even with simultaneous use of azathioprine as a steroid-sparing agent, induced flares involving debilitating fatigue, joint pain and decreases in her complement levels to below normal. Over the years, the patient had also tried plaquenil and quinacrine at various doses, but plaquenil above $100 \mathrm{mg} /$ day consistently caused the patient to experience tinnitus and ataxia, and doses of quinacrine above $100 \mathrm{mg}$ per 3 or 4 days gave the patient diarrhoea as well as yellow discolouration of the skin. The patient had, in 2012, discontinued quinacrine secondary to severe diarrhoea, and in 2013, the patient had discontinued plaquenil secondary to tinnitus. Shortly after, the patient began chloroquine $250 \mathrm{mg}$ every other day but experienced ataxia, tinnitus and heart palpitations, and subsequently discontinued the medication. In 2015, in an effort to try a new steroid-sparing medication, the patient switched from azathioprine to mycophenolate mofetil $2500 \mathrm{mg} /$ day. She continues to take low-dose prednisone at $8 \mathrm{mg} /$ day. 
The patient has a long history of photosensitivity. Shortly before the patient was hospitalised for her first SLE flare in 1983, she had been cycling for long periods of time, often in sunlight, as part of an exercise regimen. After her diagnosis, the patient initiated sun-avoidance measures, including the use of sunscreen and wearing long-sleeved clothing, which reduced her symptoms. However, the patient noticed that her photosensitivity gradually worsened over the years despite medication adjustments. She reported periods of extreme fatigue after sun exposure, and stinging and tingling of the skin immediately on exposure to the sun, as well as the development of a nonpruritic rash on her hands several days after sun exposure. Prior to these most recent episodes of photosensitivity from exposure to surgical lighting, the patient had never experienced a lupus flare from artificial light sources.

\section{INVESTIGATIONS}

A biopsy of affected skin taken during the first CLE flare in May 2011 showed interface dermatitis consistent with CLE.

\section{TREATMENT}

During both CLE flares, the patient was advised to complete a course of prednisone $60 \mathrm{mg} /$ day and taper slowly.

\section{OUTCOME AND FOLLOW-UP}

Following both occasions, the patient's rash and fatigue resolved after approximately 4 weeks. The patient continues to practice strict sun avoidance measures including wearing long-sleeved clothing, avoiding the outdoors during peak sun hours and daily use of a broad-spectrum sunscreen with high SPF. At writing, 1 year after her last CLE flare, she remains stable although symptomatic with baseline fatigue and occasional arthralgias. She continues to take prednisone $8 \mathrm{mg} /$ day and mycophenolate mofetil $2500 \mathrm{mg} /$ day.

\section{DISCUSSION}

Photosensitivity is common in SLE, with up to $73 \%$ of patients with SLE endorsing the precipitation or exacerbation of disease symptoms such as weakness, fatigue, joint pain and cutaneous lesions in response to sun exposure. ${ }^{1}$ Traditionally, photosensitivity has been understood as an unusual reaction to sunlight exposure. ${ }^{2}$ However, it is increasingly recognised that fluorescent lights can also pose as a trigger for photosensitivity in SLE. ${ }^{3}$ Fluorescent lights emit UVR, which is the component of sunlight that contributes to the disease process through pathological mechanisms involving lymphocyte recruitment and antibody-mediated cytotoxicity. ${ }^{4}$

The first report of fluorescent light as a source of photosensitivity in SLE was published in $1983 .{ }^{5}$ Since then, exposure to various fluorescent light sources has been associated with SLE or CLE exacerbations: phototherapy, tanning beds, UVA emissions from a photocopy machine and household fluorescent lamps. ${ }^{6-9}$ One study found that 13 of 30 photosensitive patients with SLE reported increases in lupus disease symptoms following fluorescent light exposure of $<1 \mathrm{~h}$ in duration. ${ }^{9}$

The clinical history and histopathological features of this case suggest that our patient developed multiple CLE flares following exposure to fluorescent lighting, specifically surgical lighting. In the literature, surgical lighting is known to be a trigger for other photosensitive diseases such as erythropoietic protoporphyria (EPP). ${ }^{10}$ Recommendations for preventing phototoxicity in patients with EPP undergoing surgery vary among centres, but many utilise protective light filters over surgical lights that block harmful wavelengths. Popular light filters include the
CLS-200-X (Madico, Inc, Woburn, Massachusetts, USA), which is a clear flexible film with good colour rendering. ${ }^{10}$ Other possible options include Dermagard (Bonwyke Ltd, Fareham, UK) and SunGard (Film Technologies Inc, St Petersburg, Florida, USA), which are both UV-protecting films. ${ }^{11}$ These light filters may also be used for extremely photosensitive lupus patients during surgical procedures, to decrease exposure to UVR. Exposure to UVR can occur through any cutaneous

\section{Patient's perspective}

- Presently, August 2015, I am still recovering from the second flare of May 2014. I am considered disabled and have not worked since May 2014. The second flare was exponentially worse than the first; following the first, it was November before I felt stable and truly able to resume my usual lifestyle again. I now appreciate concretely the fact that repeated exposures do result in significantly worsening flare activity. I am hesitant to have any dental procedure; I am seeking light shields for fluorescent lights, and a dentist or practice sensitive to the needs of persons with autoimmune disorders. I am eager to get this information about the potential for a lupus flare following a dental procedure disseminated to foster discussions related to prevention in dental and medical practices.

- Also, this flare has affected me more emotionally. I am more easily discouraged, less hopeful, feel restricted socially and 'hate' the weight I have gained! I do attend a lupus support group, and weekly individual counselling. I need to exercise more. I am on a physical and emotional roller-coaster; medication side effects and lupus 'break through' symptoms necessitate more frequent MD appointments, lab draws and medication adjustments; immunosuppressants require me to consider the risks of 'exposure' to crowds; long-term steroid use has its own set of complicating factors to manage.

- I am grateful for the medical care I am able to access with adequate medical insurance; I am grateful for the mental capacity to integrate the management of the complexity of lupus so I feel a bit in control, rather than it controlling me. I am grateful for the support of friends and family. I am grateful for the resilience of the human spirit.

\section{Learning points}

- Patients with systemic lupus erythematosus and/or cutaneous lupus erythematosus may be very photosensitive to even small amounts of ultraviolet (UV) radiation.

- Potential sources of UV radiation include sunlight, but artificial sources such as tanning beds, household fluorescent lamps and surgical lighting may also expose skin to UV rays.

- Good sun avoidance measures include wearing long-sleeved clothing, avoiding the outdoors at peak sun hours (10:00 to 16:00) and daily use of a broad-spectrum sunscreen with high SPF (preferably at least 70).

- Patients who are photosensitive to UV radiation from surgical lighting may find protective UV-blocking filters over the surgical light source to be helpful. 
surface including the oral mucosa, ${ }^{13}$ so patients may choose to utilise these filters during dental procedures as well. For everyday exposure to UVR, however, good sun avoidance measures such as wearing long-sleeved clothing, avoiding the outdoors during peak sun hours and daily use of a broad-spectrum sunscreen with high SPF, remain the mainstay. Patients who are photosensitive to artificial light sources may also consider using fluorescent light bulbs that are shielded with an extra layer of glass, also known as double-enveloped light bulbs, as opposed to unshielded single-enveloped light bulbs. ${ }^{14}$

Acknowledgements VW is supported by the Department of Veterans Affairs Veterans Health Administration, Office of Research and Development, Biomedical Laboratory Research and Development, and by the National Institutes of Health (NIH K24-AR 02207).

Contributors JT drafted the manuscript and VW revised it critically for important intellectual content. JT and VW were involved in the conception of this case report and gave final approval of the version published.

Competing interests None declared.

Patient consent Obtained.

Provenance and peer review Not commissioned; externally peer reviewed.

\section{REFERENCES}

1 Wysenbeek AJ, Block DA, Fries JF. Prevalence and expression of photosensitivity in systemic lupus erythematosus. Ann Rheum Dis 1989;48:461-3.

2 Tan EM, Cohen AS, Fries JF, et al. The 1982 revised criteria for the classification of systemic lupus erythematosus. Arthritis Rheum 1982;25:1271-7.
3 Klein RS, Sayre RM, Dowdy JC, et al. The risk of ultraviolet radiation exposure from indoor lamps in lupus erythematosus. Autoimmun Rev 2009;8:320-4.

4 Kim A, Chong BF. Photosensitivity in cutaneous lupus erythematosus. Photodermatol Photoimmunol Photomed 2013; 29:4-11.

5 Martin L, Chalmers IM. Photosensitivity to fluorescent light in a patient with systemic lupus erythematosus. J Rheumatol 1983;10:811-12.

6 Dowdy MJ, Nigra TP, Barth WF. Subacute cutaneous lupus erythematosus during PUVA therapy for psoriasis: case report and review of the literature. Arthritis Rheum 1989:32:343-6.

7 Stern RS, Docken W. An exacerbation of SLE after visiting a tanning salon. JAMA 1986:255:3120.

8 Klein LR, Elmets CA, Callen JP. Photoexacerbation of cutaneous lupus erythematosus due to ultraviolet $\mathrm{A}$ emissions from a photocopier. Arthritis Rheum 1995:38:1152-6.

9 Rihner M, McGrath H Jr. Fluorescent light photosensitivity in patients with systemic lupus erythematosus. Arthritis Rheum 1992;35:949-52.

10 Wahlin S, Srikanthan N, Hamre B, et al. Protection from phototoxic injury during surgery and endoscopy in erythropoietic protoporphyria. Liver Transpl 2008;14:1340-6

11 Fenton L, Dawe R, Ibbotson S, et al. Impact assessment of energy-efficient lighting in patients with lupus erythematosus: a pilot study. Br J Dermatol 2014;170:694-8.

12 Eadie E, Ferguson J, Moseley H. A preliminary investigation into the effect of exposure of photosensitive individuals to light from compact fluorescent lamps. $\mathrm{Br} J$ Dermatol 2009;160:659-64.

13 Breger J, Baeva L, Agrawal A, et al. UVB-induced inflammatory cytokine release, DNA damage and apoptosis of human oral compared with skin tissue equivalents. Photochem Photobiol 2013;89:665-70.

14 Moseley $\mathrm{H}$, Ferguson J. The risk to normal and photosensitive individuals from exposure to light from compact fluorescent lamps. Photodermatol Photoimmunol Photomed 2011;27:131-7.

Copyright 2015 BMJ Publishing Group. All rights reserved. For permission to reuse any of this content visit http://group.bmj.com/group/rights-licensing/permissions.

BMJ Case Report Fellows may re-use this article for personal use and teaching without any further permission.

Become a Fellow of BMJ Case Reports today and you can:

- Submit as many cases as you like

- Enjoy fast sympathetic peer review and rapid publication of accepted articles

- Access all the published articles

- Re-use any of the published material for personal use and teaching without further permission

For information on Institutional Fellowships contact consortiasales@bmjgroup.com

Visit casereports.bmj.com for more articles like this and to become a Fellow 